\title{
Correspondence Between Constructive Real Numbers and $L$ Number in Constructive Mathematics
}

\author{
Xin Shen ${ }^{1 *}$, Yiding Huang ${ }^{2}$, Shixiang Wang ${ }^{3}$ \\ ${ }^{I}$ Nanjing Foreign Language School, Nanjing, China \\ ${ }^{2}$ Beijing 101 middle school, Beijing, China \\ ${ }^{3}$ Oxford International College, Oxford, OX4 1BD, United Kingdom \\ *Corresponding author. Email: 2830627975@qq.com
}

\begin{abstract}
In a broad sense, construction real number is a constructable real number, relative to the set of some construction methods. This essay mainly focuses on the left numbers in constructive analysis. After introducing the basic context and conditions in Constructive Mathematics, connections between concepts are put into consideration and discovered with reference. Specific propositions are also discussed and utilized to simplify and prove the statements listed below. It is deduced that in the context of constructive mathematics, there exists an algorithm such that given any constructive real number, it produces the corresponding Left $(\mathrm{L})$ number, given the presumption that with equivalent constructive real numbers (CRN), equivalent Left numbers can be the output. In addition, the Specker sequence is utilized to prove that the converse statement is false. It is hoped that the paper can introduce more people to basic concepts and application of theorems in analysis.
\end{abstract}

Keywords: Specker sequence, constructive mathematics, Specker Sequence

\section{INTRODUCTION}

Left number is a sequence of increasing rational numbers, which can be derived by some algorithm and is bounded above by a rational number. Every rational number can be thought of as a left number given as a geometric series of rational numbers. We focus on the correspondence between constructive real numbers and Left(L) numbers.

In this essay, some definitions in the constructive mathematical context are introduced and clarified including algorithm, constructive real number, decidable, and enumerable sets. Then, it is proved that there is an algorithm that given any constructive real number produces the corresponding left number. It is also observed that the Specker sequence example shows that the converse statement is false. With further observation and deduction, it is logical to divide the proof of these two statements into three parts: 1) finding an algorithm using standard regulator to get an increasing sequence of rational numbers 2) proving that equivalent $\mathrm{CRN}$ give equivalent left numbers. 3) providing and explaining an example of a left number which does not converge to CRN. It is hoped that through research and proof of the above problems, the paper can provide some ideas for those who are interested in topology. In case that constructive mathematics is still unknown to most people, here are some information in the context of constructive mathematics to further enhance comprehension and understanding:

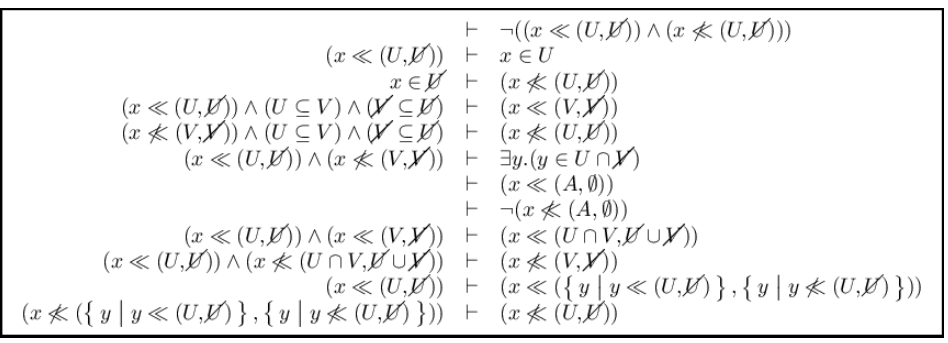

Figure 1 Linear logic for constructive mathematics 
What is the relationship between constructive mathematics and classical mathematics? The relationship is clear as follows:

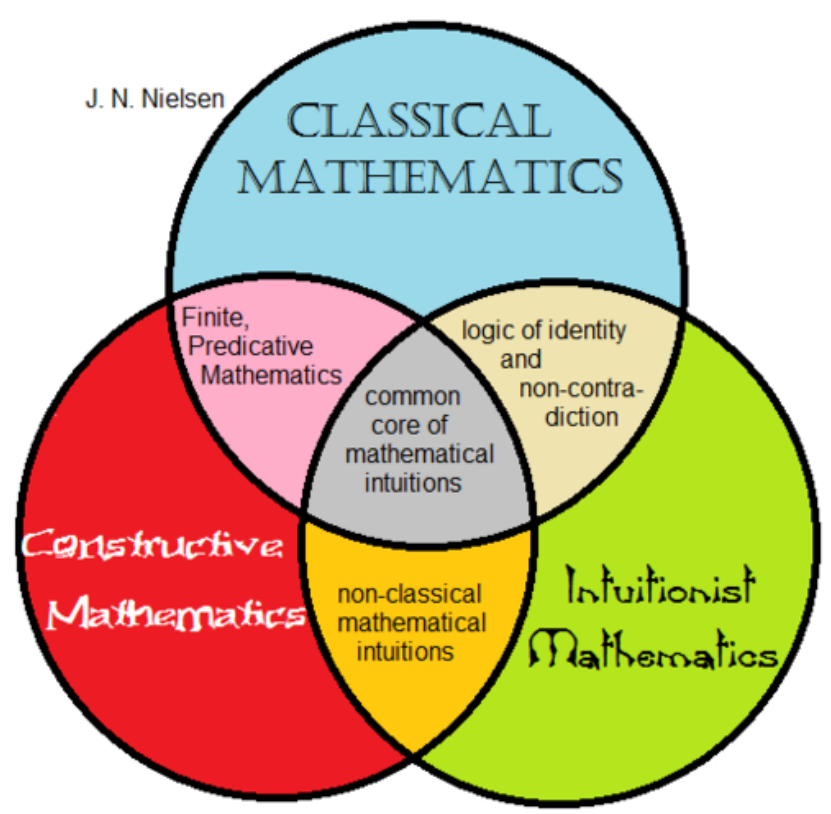

Figure 2 Is mathematics this whole structure - classicism, constructivism, and intuitionism taken together - plus whatever else is done in the name of mathematics?

\section{DEFINITIONS}

\subsection{L(left) number}

Left number is any strictly increasing algorithmically given sequence of rational numbers bounded from above by a bounding element $\theta$, which is a rational number [1].

\subsection{Computable (constructive) real number with Stantard Regulator}

There are two similar definitions that are equivalent:

There exists a computable function which, given any positive rational error bound $\varepsilon$, produces a rational number $r$ such that $|r-a| \leq \varepsilon[2]$.

There is a computable sequence of rational numbers $q_{i}$ converging to a such that

$$
\left|q_{i}-q_{i+1}\right|<2^{-i} \text {, for each i [3]. }
$$

\subsection{Computably Enumerable set}

A set $\mathrm{S}$ of natural numbers is called computably enumerable if there is a partial computable function whose domain is exactly $\mathrm{S}$, meaning that the function is defined if and only if its input is a member of S [4].

\subsection{Decidability}

There exists a general recursive function fsuch that $\mathrm{M}=\{\mathrm{n}: \mathrm{f}(n)=0\}$. In this case $\mathrm{f}$ is an algorithm for checking whether a natural number belongs to $\mathrm{M}$ :

indeed, $\mathrm{n} \in M$ is equivalent to $\mathrm{f}(n)=0$. A decidable set of natural numbers is also often called a general recursive set or a recursive set.

\subsection{Algorithm}

In mathematics, an algorithm is a process that describes a series of steps that may be used to perform a mathematical computation.

\section{PROOF}

FIRST LET'S ASSUME THAT CONSTRUCTIVE REAL NUMBERS HAVE A STANDARD REGULATOR.

Let the CRN have a sequence $\alpha(n)$ equipped to the standard regulator. There exist an algorithm that transform the sequence into $\mathrm{x}$, such that

$$
x(n)=\alpha(n+1)-\frac{1}{2^{n}}
$$

In order for this sequence to be increasing, we should have:

$$
\alpha(n+1)-2^{-n}<\alpha(n+2)-2^{-(n-1)}
$$




$$
\alpha(n+1)-\alpha(n+2)<2^{-(n+1)}
$$

Now we prove that this is true.

From the definition of standard regulator, we know

$$
\forall n, l \geq n,|\alpha(k)-\alpha(l)|<2
$$
have

Substitute $n$ with $n+1$, $k$ with $n+1$, and 1 with $n+2$, we

$$
|\alpha(n+1)-\alpha(n+2)|<2^{-(n+1)}
$$

which tells us that

$$
\alpha(n+1)-\alpha(n+2)<2^{-(n+1)}
$$

Therefore, the sequence is strictly increasing.

The sequence is also bounded above by $\alpha(1)+\frac{1}{2}$. Therefore, we know that $\mathrm{x}$ is a left number.

Moreover, from the definition of $\mathrm{CRN}$, which is provided above,

$$
x(n+1)-x(n)=\alpha(n+1)-\alpha(n)+\frac{1}{2^{n+1}}
$$

which is greater than 0

So, from this we can generate a Left number $\mathrm{x}$.

Let's first define the comparison between Left numbers:

if $L_{1}<L_{2}$, then there exists a number in the sequence $L_{2}$ that is greater than every number in $L_{1}$

Moreover, let's define the equivalence of two $\mathrm{L}$ numbers:

$$
\text { if } L_{1} \leq L_{2} \text { and } L_{1} \geq L_{2} \text {, then } L_{1}=L_{2}
$$

To simplify, we use $\alpha(n)$ and $\alpha^{\prime}(n)$ as two different sequences which converge are equivalent.

Let $\mathrm{L}$ number $x(n)$ be $\alpha(n)-2^{-n}$ and $\mathrm{L}$ number $y(n)$ be $\alpha^{\prime}(n)-2^{-n}$

So we have

$$
\begin{gathered}
\left|\alpha(n)-\alpha^{\prime}(n)\right|<2^{-n} \\
\text { s }|x(n)-y(n)|<2^{-n}
\end{gathered}
$$

Assume $x \leq y$ is false, then there exists a $\mathrm{n}$ such that for any i, $x(n)>y(i)$. Moreover, there exists a $\mathrm{k}$, such that $\mathrm{k}>\mathrm{n}$ and $x(n+1)-x(n)>2^{-k}$.

Moreover, since $\mathrm{x}(\mathrm{n})$ and $\mathrm{y}(\mathrm{n})$ strictly increases, we get

$$
\begin{gathered}
|x(k)-y(k)|=x(k)-y(k) \geq x(n+1)-y(k)> \\
x(n+1)-x(n)>2^{-k}
\end{gathered}
$$

Moreover, since

$$
|x(n)-y(n)|<2^{-n}
$$

Substitute $\mathrm{n}$ with $\mathrm{k}$, we have

$$
|x(k)-y(k)|<2^{-k}
$$

This is a contradiction. Therefore, equivalent $\mathrm{L}$ numbers can be outputted when the input CRNs are equivalent.

To prove this, we can simply look for an example of left number that does not converge to CRN.
Let's first show that the problem is equivalent to finding as Specker sequence:

From the definition of Specker sequence, we know that the sequence is strictly increasing, has limit, and its supremum cannot be computed, which means that it's not a CRN.

Therefore, Specker sequence is Left number, and it has $\mathrm{CRN}$ which requires both a series and regulator.

Here is an example of the generating of Specker sequence [5]:

Consider recursively enumerable set of natural numbers, A, and it is not decidable, and $a_{i}$ represents a kind of computable enumeration of A. It does not contain any form of repetition. Next, let's define a sequence of rational numbers, call it $q_{n}$.

$$
\mathrm{q}_{n}=\sum_{i=0}^{n} 2^{-a_{i}-1}
$$

Each $q_{n}$ is rational and nonnegative, and as a sequence, $q_{n}$ is strictly increasing. Moreover, estimating each $q_{n}$ against the series is reasonable, because the kind of computable enumeration of $A$ has no repetition.

$$
\sum_{i=0}^{\infty} 2^{-i-1}=1
$$

Therefore, it is clear that the sequence $q_{n}$ is bounded above by 1 , a rational number.

With this in mind, we know that $q_{n}$ has a supreme, satisfying one of the key propositions of Specker Sequence.

Plus, since it is known that $x$ is not a computable real number, if $x$ were computable then let's assume that there existed a computable function $\mathrm{r}(\mathrm{n})$ such that $\left|q_{j}-q_{i}\right|<$ $\frac{1}{n}$, with condition $i, j>r(n)$. After comparing the binary expansion of $\mathrm{x}$ with the binary expansion of $q_{i}$ for increasing values of $I$, it is logical to compute $r$. Consider the definition of $q_{i}$. Each time i increases by 1 , there is a single binary digit that goes from 0 to 1 . See the pattern below.

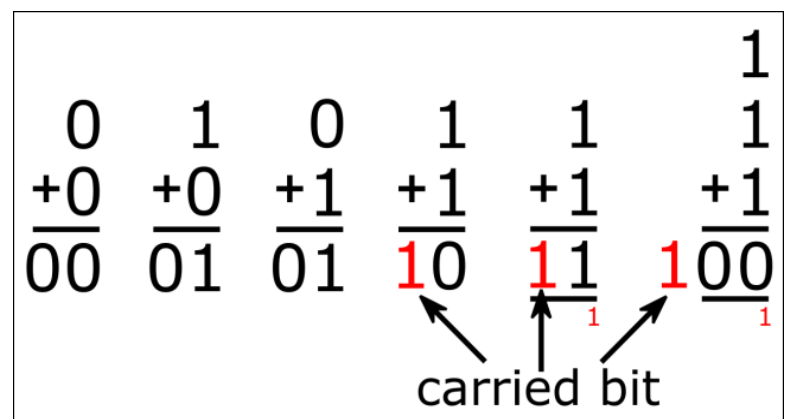

Figure 3 Property of binary numbers

There will be, therefore, an $\mathrm{n}$ such that a very large initial segment of $\mathrm{x}$ is already judged by $q_{n}$. No additional binary digits in that part could be transformed 
again, leading to a calculation of the value of the distance between $q_{i}$ and $q_{j}$ for $\mathrm{i}, \mathrm{j}>\mathrm{n}$.

If $\mathrm{k}$ will be enumerated into $a_{i}$, it must be done for a value of $i$ less than $r\left(2^{k+1}\right)$, leaving a finite number of possibilities in which $\mathrm{k}$ can be enumerated. It is always important to check and return 0 or 1 depending on where $\mathrm{k}$ is found.

Therefore, if there is an input $\mathrm{k}$, we can compute $\mathrm{r}\left(2^{\mathrm{k}+1}\right)$. Although it is possible that $\mathrm{k}$ appears in the sequence $a_{i}$, which would make the sequence $q_{i}$ increase by $2^{-\mathrm{k}-1}$, this remains impossible if all elements of $q_{i}$ are within $2^{-\mathrm{k}-1}$ of each other.

For computable analysis, the existence of Specker sequences has consequences. Another example is provided here, in case that the previous one is too complicated to understand:

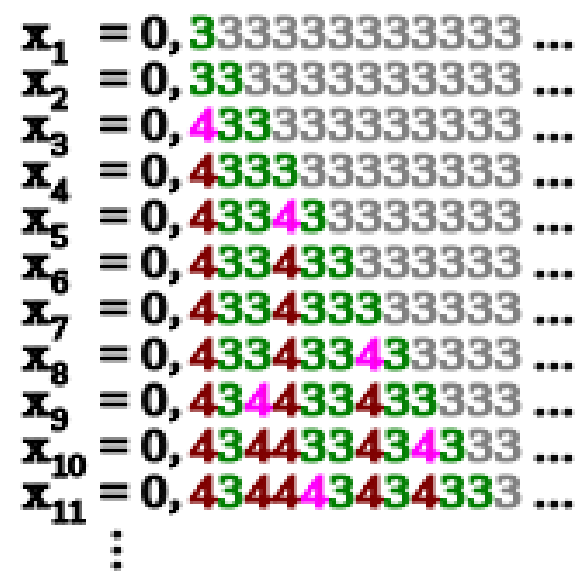

Figure 4 A Specker sequence. The nth digit of $x_{k}$ is 4 if $\mathrm{n} \leq \mathrm{k}$ and the computation of $\{\mathrm{n}\}(\mathrm{n})$ halts after $\mathrm{k}$ steps; otherwise it is 3 .

\section{CONCLUSION}

This paper investigates the correspondence between constructive real numbers and 1 number in the context of constructive mathematics. It is proved that equivalent CRN give equivalent left numbers, there is an algorithm that given any constructive real number produces the corresponding left number, and that the converse statement is false. The paper focuses on finding an appropriate sequence to make connections between constructive real numbers and Left number. Besides, Specker Sequence is cleverly cited as a counterexample of a left number that does not correspond to a constructive real number. The paper aims to introduce more people to fundamental concepts and principles in constructive mathematics and analysis and clarify underlying relations between different concepts. The relationships may be hard to observe, but with patience and logical deduction from authoritative propositions, people can discover interesting underlying relations, contributing to a more intact and deductible mathematical system with the context of constructive mathematics.

\section{ACKNOWLEDGMENT}

This paper is finished under the guidance of Professor Vladimir Chernov, from Dartmouth University. We are sincerely grateful for his encouragement and support, which helped build up basic structures and revised small errors. Also, thanks are given to Neoscholar for organizing the program on which the research was done. Plus, It is noted that the problem of deducing relationships and deciding whether the statement is applicable in the context of constructive mathematics, which we are solving, was created because of collaboration of Viktor Chernov and Vladimir Chernov.

\section{REFERENCES}

[1] Errett Bishop (Author), Michael Beeson (Introduction), Foundations of Constructive Analysis, Ishi Pres, 2012

[2] wikipedia. (2020) Computable_number https://en.wikipedia.org/wiki/Computable_number

[3] A. Shen, and N. Vereshchagin, Nikolai Konstantinovich, Computable Functions, Amer Mathematical Society, 2002.

[4] Peter Cholak. (2006) The Computably Enumerable Sets. https://www3.nd.edu/ cholak/papers/china.pdf

[5] Kushner, B. A, Lectures on Constructive Mathematical Analysis, American Mathematical Society, 1984. 